\title{
Repair of severe traumatic nasal alar defects with combined pedicled flap and conchal cartilage composite grafts: a retrospective study
}

\author{
Zhenyu Zhang ${ }^{1 \#}$, Lihui Cheng ${ }^{2 \#}$, Tony Chieh-Ting Huang ${ }^{3}$, Hua Hu ${ }^{1}$, Ruiqi Liu ${ }^{1}$, Yi Pu ${ }^{1}$, Ru Wang ${ }^{1}$, \\ Zhengyong Li ${ }^{1}$, Junjie Chen ${ }^{1}$, Ying Cen ${ }^{1}$, Guopeng Liang ${ }^{4}$, Yong Qing ${ }^{1}$
}

${ }^{1}$ Department of Aesthetic Plastic and Burn Surgery, West China Hospital, Sichuan University, Chengdu, China; ${ }^{2}$ Department of Central Sterile Supply, West China Hospital, Sichuan University, Chengdu, China; ${ }^{3}$ Division of Plastic Surgery, Mayo Clinic, Rochester, MN, USA; ${ }^{4}$ Department of Critical Care Medicine, West China Hospital, Sichuan University, Chengdu, China

Contributions: (I) Conception and design: Z Zhang, G Liang, Y Qing; (II) Administrative support: Y Cen, J Chen; (III) Provision of study materials or patients: Z Zhang, L Cheng, Y Qing; (IV) Collection and assembly of data: Z Zhang, L Cheng, H Hu; (V) Data analysis and interpretation: Z Zhang, L Cheng, R Liu, G Liang; (VI) Manuscript writing: All authors; (VII) Final approval of manuscript: All authors.

\#These authors contributed equally to this work.

Correspondence to: Guopeng Liang. Department of Critical Care Medicine, West China Hospital, Sichuan university, No. 37 Guo Xue Xiang, Wuhou District, Chengdu, China. Email: liangguopeng007@163.com; Yong Qing. Department of Aesthetic Plastic and Burn Surgery, West China Hospital, Sichuan University, No. 37 Guo Xue Xiang, Wuhou District, Chengdu, China. Email: 28840535@qq.com.

Background: Repair of traumatic alar defect is challenging because poor blood supply is caused by contracture scars, which sometimes extend beyond the alar groove. However, few studies have investigated the reconstruction results of severe traumatic cases. This study aimed to examine the clinical outcomes of severe traumatic alar defect reconstruction using either pedicled nasolabial or forehead flaps combined with conchal cartilage.

Methods: This retrospective study investigated the clinical characteristics and treatment effects of 17 patients with severe traumatic alar defects treated in a single plastic surgery center from March 1, 2015, to September 1, 2018. All cases were scored and graded with regard to the size and depth of the alar defect and the surrounding scar according to the Alar Defect Severity Score (ADSS). Surgical outcomes were evaluated on the basis of the severity of defect before repair, donor site distortion, and postoperative nasal symmetry, especially shape and color.

Results: The average ADSS of the cases was $8.1 \pm 0.8$ (highest score, 9.0). No flap necrosis or any complications were observed postoperatively. The symmetry of the bilateral alae was satisfactory. No color distinction between grafts and surrounding tissues, retraction, or inferior displacement of the ala was observed at an average follow-up of $24.2 \pm 10.4$ months (range, $8-42$ months). The average postoperative surgeon-based evaluation score was $4.3 \pm 0.2$ (highest score, 5.0). Esthetic and functional results were satisfactory in all cases.

Conclusions: A pedicled flap combined with conchal composite grafts should be considered for the treatment of severe traumatic alar defect. This is a reproducible technique that enables a predictably decent outcome for severe traumatic alar defect, especially in Asian patients.

Keywords: Nasal ala; nasolabial flap; forehead flap; conchal cartilage; traumatic alar defect

Submitted Aug 28, 2020. Accepted for publication Oct 30, 2020.

doi: $10.21037 / \mathrm{atm}-20-6454$

View this article at: http://dx.doi.org/10.21037/atm-20-6454 


\section{Introduction}

The nasal ala, which is a cosmetically sensitive area, is characterized by thick and sebaceous skin, thin and strong supporting cartilage, a free margin, and delicate bilateral symmetry both in size and color (1). Satisfactory repair of nasal alar defect has always been one of the most challenging problems for plastic surgeons.

Traumatic alar defects are typically caused by animal bite, traffic accident, burn, and injury from nasal hemangioma treatment. Unlike more common clinical cases caused by oncologic resection, which typically occur in older patients, the incidence of traumatic types is higher in children and inevitably leads to alar dysplasia. Furthermore, the initial treatment of traumatic alar injuries is often inadequate, leading to a high rate of scarring. As a result, when these patients seek medical consultation as adults, the degree of alar defect with contracture is often so severe that they can extend beyond the alar groove, and significant disproportion is commonly present. Because it is frequently confused with defects following tumor resection, the number of reported cases of traumatic nasal alar defect is extremely limited and, subsequently, effective treatment approaches have been underexplored, especially in severe cases $(2,3)$.

Although a free auricular composite flap is commonly used to restore full-thickness alar defects, soft tissue atrophy and pigmentation habitually occur during later stages due to poor vascularity from surrounding tissues, thus restricting its application in large area defects $(3,4)$. In addition, partial auricular excision can cause mismatch in the bilateral size and shape of the ears. Therefore, repair of severe traumatic nasal alar defect requires not only composite tissue graft but also sufficient blood supply to ensure graft survival relative to oncologic resection defects $(5,6)$. Forehead and nasolabial flaps are the preferred mediums of nasal repair over other flaps because of their stable blood supply, sufficient skin, and ease of access $(7,8)$. Conchal cartilage is frequently used in rhinoplasty because of its similar texture to alar cartilage and the size and supporting structure of the external ear can be preserved.

This study aimed to analyze and present the results of pedicled nasolabial or forehead flap combined with conchal cartilage in the reconstruction of full-thickness traumatic alar defects.

We present the following article in accordance with the STROBE reporting checklist (available at http://dx.doi. org/10.21037/atm-20-6454).

\section{Methods}

A total of 17 patients (male, 10; female, 7; mean age, 29 years; range, 19-42 years) who underwent nasal alar reconstruction from March 1, 2015, to September 1, 2018, were analyzed retrospectively. The inclusion criteria included $\geq 18$ years of age, range of defect was limited to the ala without influencing other subunits of the nose, and no nasal reconstructive surgery prior to this treatment. The exclusion criteria were $\leq 17$ years old, defect involving structures outside the ala, and rhinoplasty previous to this treatment. Informed consent was provided by all patients preoperatively. The study was approved by the Clinical Research Ethics Committee of West China Hospital of Sichuan University. All procedures performed in this study involving human participants were in accordance with the Declaration of Helsinki (as revised in 2013). All traumatic alar defects were scored with regard to size, depth, and surrounding scar according to the Alar Defect Severity Score (ADSS) (Table 1). The defects were graded as mild, moderate, or severe.

\section{Surgical technique}

Traumatic alar repair using a forehead flap included three stages: tissue expansion, reconstruction, and pedicle amputation. The first and third procedures were performed under local anesthesia using $1 \%$ lidocaine with 1:200,000 epinephrine, while the second procedure was done under general anesthesia. A $100 \mathrm{~mL}$ tissue expander (Soft Tissue Expander; Shanghai Dongyue Medical Health Product Co., Ltd., Shanghai, China) was implanted subcutaneously and gradually expanded to adequate size over 6-8 weeks (Figure 1). In the second procedure, the nasal alar scar was resected and contracture tissue was fully released. The remaining alar skin was cut along the marking line to form a flap; then, the flaps were rotated, with the defective edge as the axis. These two reversed flaps were sutured to partly rebuild the mucosal layer of the nostril, with the skin facing inward and the wound surface facing outward. The skin and mucosa layers surrounding the defect were separated to facilitate the insertion of composite cartilage tissue. The wounds were thoroughly washed, and no active bleeding was observed. Then, cartilage and anterior skin from the conchal cavity were harvested to accommodate the size of defect, as measured after scar excision. The conchal donor defect was covered by a full-thickness skin graft or posterior auricular random skin flap. Proper compression dressing was 
Table 1 Traumatic Alar Defect Severity Score and grade standard

\begin{tabular}{lccc}
\hline Characteristics & \multicolumn{3}{c}{ Alar Defect Severity Score (ADSS) } \\
\cline { 2 - 4 } & 1 & 2 & 3 \\
\hline Defective size $\left(\mathrm{cm}^{2}\right)$ & $\leq 1.5$ & $>1.5$ and $<3.5$ & Full thickness \\
Defect involving & Skin & Skin and subcutaneous tissue with intact mucosa & Contracted \\
Surrounding scar & Slight & Scar with pigment difference & \\
\hline
\end{tabular}

ADSS: $\leq 2$ (mild); between 3 to 6 (moderate); $\geq 7$ (severe).

applied to avoid local hematoma and ensure graft survival. Compound tissue flap was inset and sutured to the defective ala using PDS 5-0 sutures (Ethicon Inc. Somerville, NJ, USA) to restore the mucosal and cartilaginous layers (Figure 1). Then, the expanded pedicled forehead flap was designed and rotated $180^{\circ}$ around the supertrochlear artery to cover the cartilage and reconstruct the alar rim. The flap was sutured to the skin margin of the alar defect using 6-0 PDS. Tension-free wound closure could then be easily performed in the forehead donor site.

The application of the nasolabial flap to restore the ala was divided into two stages: defect repair was performed first, followed by pedicle amputation. The nasolabial flap pedicled on the cranial side was raised and rotated to the alar side to reconstitute the skin layer and alar rim. The donor area was closed directly in a side-to-side manner. A silicone tube was placed inside the nostril of all patients for support and removed with the stitches on postoperative day 14. Pedicle amputation was implemented on the premise of graft survival 4 weeks after the reconstructive procedures, and the excess pedicle skin was used to cover any defects caused by scar removal around the alar groove (Figure 1).

\section{Postoperative effect evaluation}

Surgical outcomes were evaluated on a 5-point Likert scale (1, least favorable; 2 , bad; 3 , moderate; 4 , favorable; 5 , most favorable). Postoperative pictures were evaluated by three plastic surgeons who were not involved in the surgeries and blinded to the surgical interventions. According to the published literature and the clinical characteristics of our patients, evaluation criteria included the severity of defect before repair, donor site distortion, and postoperative nasal symmetry, particularly shape and color $(2,3)$. All patients underwent routine outpatient follow-up examination after the operation.

\section{Statistical analysis}

The data of ADSS, follow-up time and postoperative evaluation score were recorded and analyzed by Microsoft Excel (Microsoft office 2019, Microsoft, USA).

\section{Results}

In all 17 patients, the size of defects ranged from $1.7 \mathrm{~cm}$ $\times 2.0 \mathrm{~cm}$ to $2.1 \mathrm{~cm} \times 2.6 \mathrm{~cm}$, and the average size was $1.9 \mathrm{~cm} \times 2.4 \mathrm{~cm}$. No infection, partial or total losses, venous insufficiency in the flap, or any complications were observed in any of the cases. The ADSSs of all patients were $\geq 7$ (i.e., severe). The average ADSS of cases was $8.1 \pm 0.8$ (highest score, 9.0). The patient's details are presented in Table 2.

The symmetries of the bilateral alae were satisfactory, and none of the cases had color mismatch between grafts and surrounding tissues. All reconstructed alae were smoothly integrated with surrounding tissues. No distortions or hypertrophic scars were observed in the donor sites. A slight nasolabial scar appeared in a patient, but she did not consent to any further intervention. No retraction or inferior displacement of the ala was observed at an average follow-up of $24.2 \pm 10.4$ months (range, $8-42$ months). The average postoperative surgeon-based evaluation score was $4.3 \pm 0.2$ (highest score, 5.0), and the esthetic and functional results were satisfactory in all patients (Figures 2,3).

\section{Discussion}

This retrospective study evaluated the results of pedicled nasolabial or forehead flap combined with conchal cartilage in the reconstruction of full-thickness traumatic alar defects. The pedicled flap combined with conchal composite grafts had satisfactory results. None of the patients experienced flap necrosis, postoperative complications, color inconsistency between graft and surrounding 

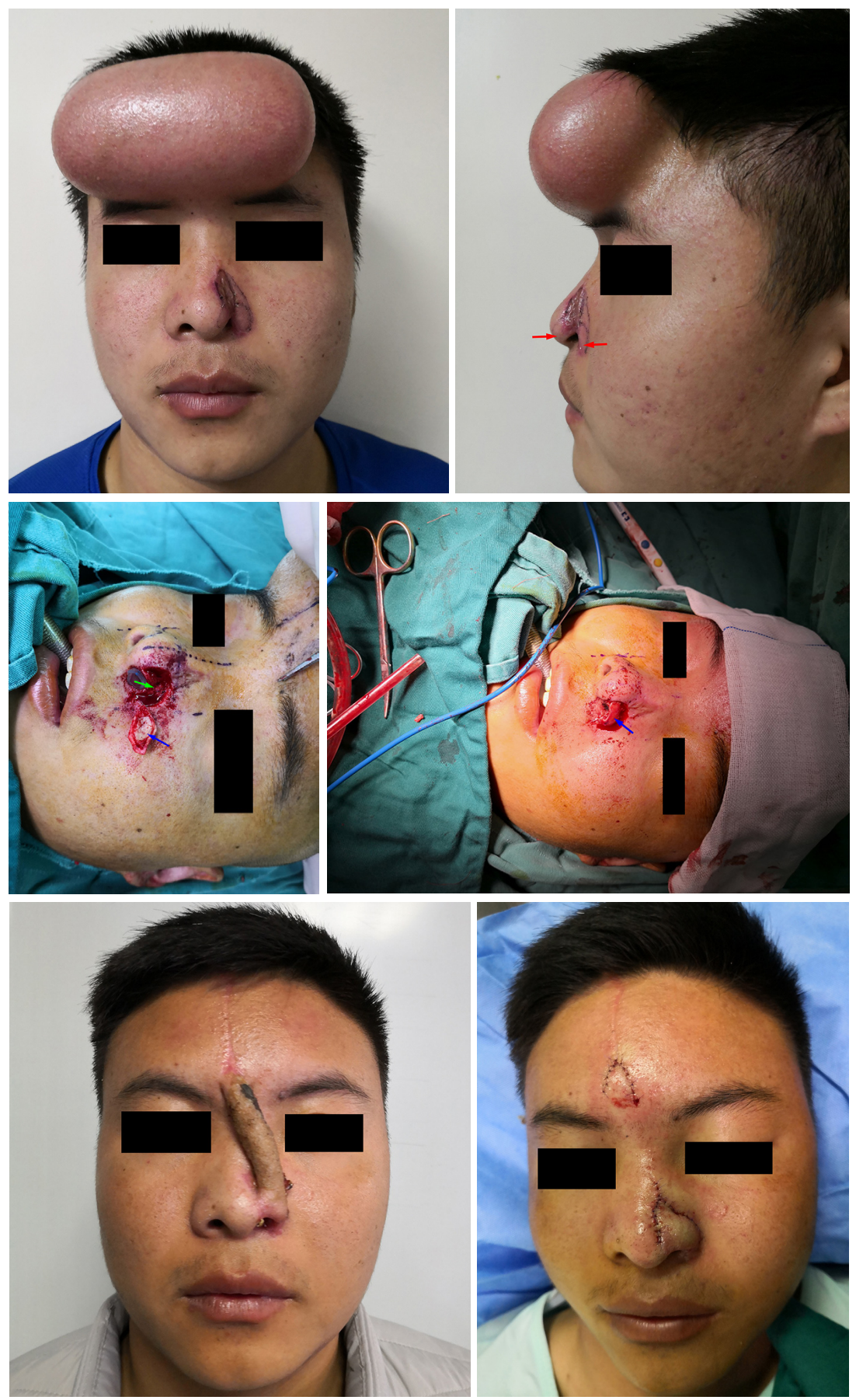

Figure 1 A 31-year-old man with a left alar defect due to a traffic accident that occurred 9 months before repair. After the injury, only hemostasis of the wound had been performed. The wound healed with surrounding tissue growth, and a hypertrophic scar formed over the defect. A tissue expander was implanted during the first-stage operation, which gradually expanded to adequate size in 7 weeks. Frontal (top left) and lateral (top right) views and preoperative design before the second-stage operation. The remaining alar skin was cut along the marking line to form a flap (red arrow), then the flaps were rotated with the defect edge as the axis. These two reversed flaps were sutured to partly rebuild the nostril mucosal layer of (green arrow). The alar scar was removed (middle left), and composite cartilage grafts (blue arrow) were harvested and sutured to the defect region (middle right). Frontal view before (bottom left) and after (bottom right) pedicle amputation and removal of residual scars. 
Table 2 Summary of patients' data

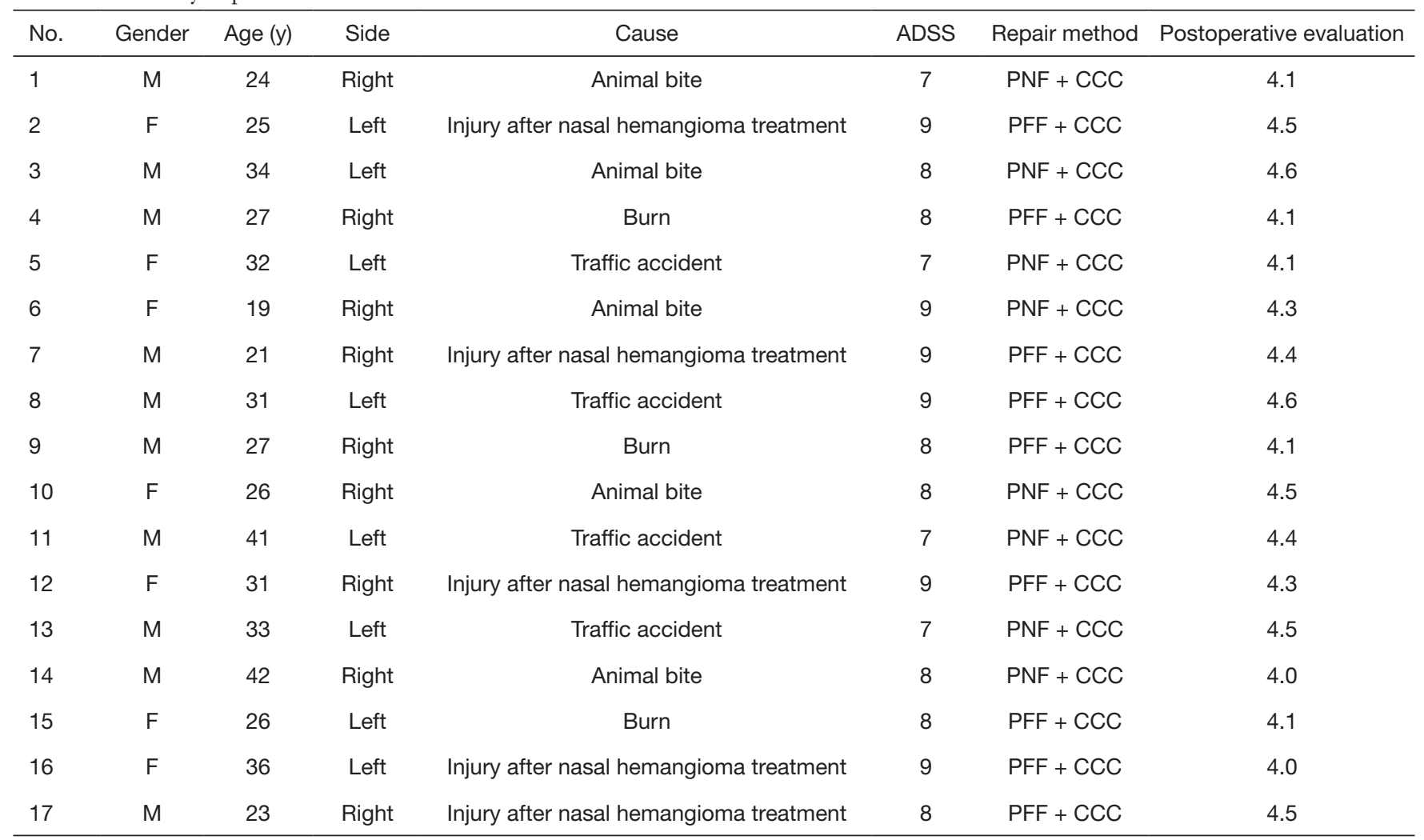

PNF, pedicled nasolabial flap; PFF, pedicled forehead flap; CCC, conchal cartilage composite.

tissues, retraction, or inferior displacement of the ala. The symmetry of the bilateral alae was satisfactory, as were the esthetic and functional results.

The use of adjacent skin and cartilage tissues to reconstruct the nasal ala has been proven to be more conducive to achieving good esthetic results (3). Typically, the dermis is thicker and more compact in Asians than in Europeans, and this leads to rounder and thicker alae (9). Moreover, matching skin texture and color are more essential in Asian patients (10). Therefore, the forehead and nasolabial flaps are suitable for restoration of severe traumatic nasal alar defects.

The most conspicuous differences between traumatic and oncologically resected alar defects are the extension of the surrounding contracture scar beyond the alar groove and the poor vascularization of the recipient bed in the traumatic cases. Excision of visible scars together with total release of contracture tissue is beneficial in the reconstruction of a smooth and natural transition between the skin flap and donor site. After scar removal and incision margin repair, the resulting defect area is generally larger than preoperative measurements. Thus, the use of pedicled flaps with sufficient tissues, such as forehead and nasolabial, is the preferred strategy for the reconstruction of traumatic alar defects. Owing to adequate and stable blood supply, pedicled flaps can ensure the survival of free transplanted cartilage and avoid postoperative ischemic necrosis of the graft, which causes atrophy and color inconformity. Compared with bilobed or trilobed flaps, a nasolabial flap is advantageous because it is less disruptive to nasal and facial tissues and produces a less visible scar on the nasal dorsum $(11,12)$. The high tension and small surface area of skin of the nasal dorsum make it more difficult to use a dorsal flap for alar repair in Asian patients (13). Moreover, a horizontal advancement flap is less preferable for the repair of large defects, as it has a higher chance of distorting the nasal contour and alar rim (14). Because the previous trauma could destroy the blood vessels around the alar, the repair method which needs vascular anastomosis was not considered as the first choice of treatment in this group of patients. In addition, too complex and meticulous operation is not conducive to wide application, because similar 


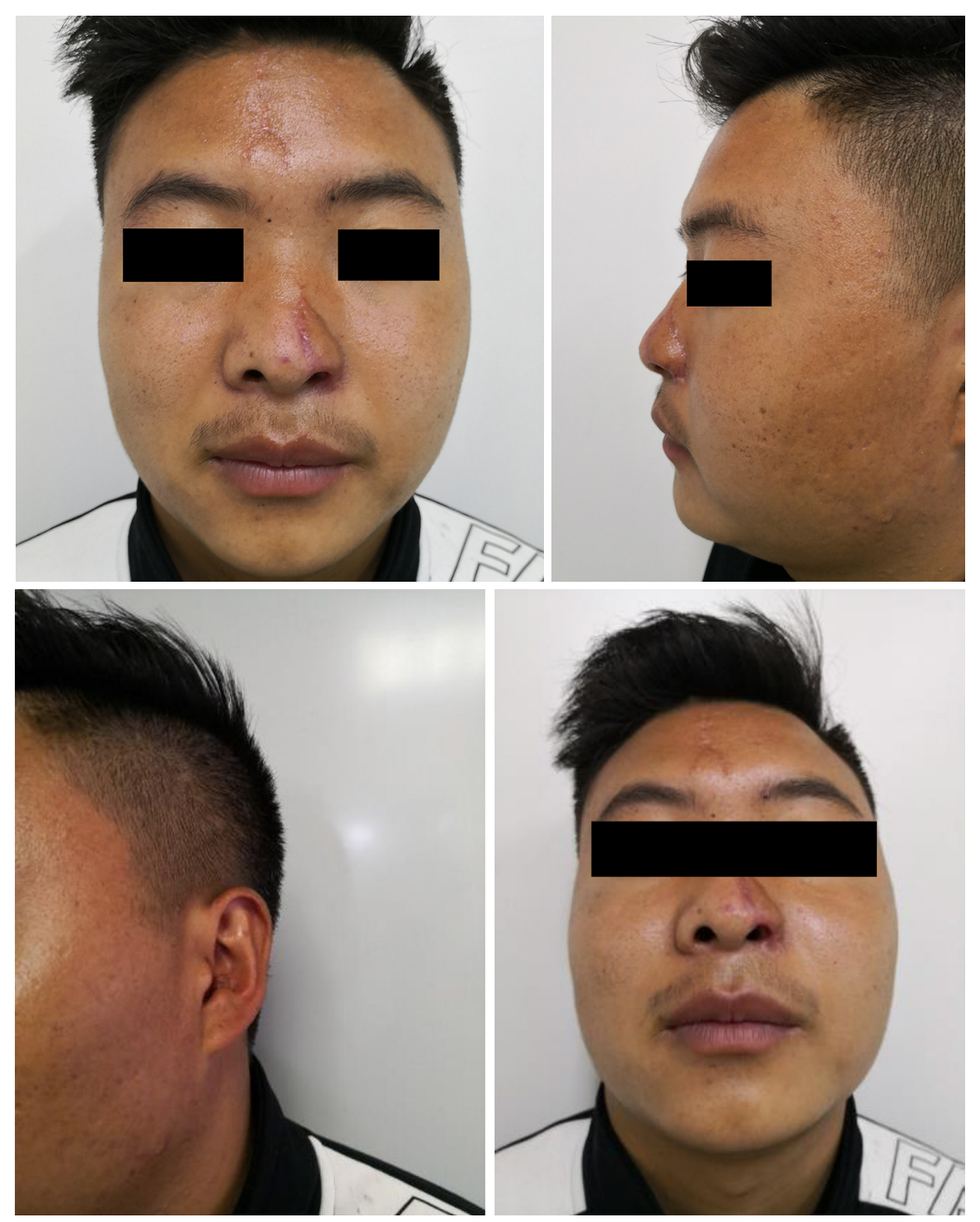

Figure 2 Reconstructive effects in the ala (top and bottom right) and donor ear (bottom left) 12 months after the reconstructive operation.

postoperative effects could be achieved through simpler and easier techniques.

Generally speaking, an expanded pedicled forehead flap can cover a larger defect compared with a nasolabial flap, which makes it more suitable for patients wanting to avoid visible scars in the cheek area. Almost all the patients in this study with an ADSS of 9 underwent the expanded pedicled forehead flap procedure. The forehead flap is usually our preferred approach for cases with extensive scars near the nasolabial region due to burn or injury. Because the expanded pedicled flap sufficiently covered large areas, the skin surrounding the defective part could be applied to rebuild the inner layer, which effectively lessened the ear donor site damage, despite the relatively larger skin defect area. Although scars in the forehead region can be concealed by hair, for patients who cannot tolerate prolonged tissue expansion, the nasolabial flap is the preferred method for reconstructing the defective ala. Rather than a single-stage operation, flap pedicle division in the second stage was conducive to retaining both the nasofacial angle and the natural nasal contour, especially in larger defects (15). In addition, the extra skin from the pedicle could be used to cover the secondary wounds created by removal of scarring beyond the alar groove. 

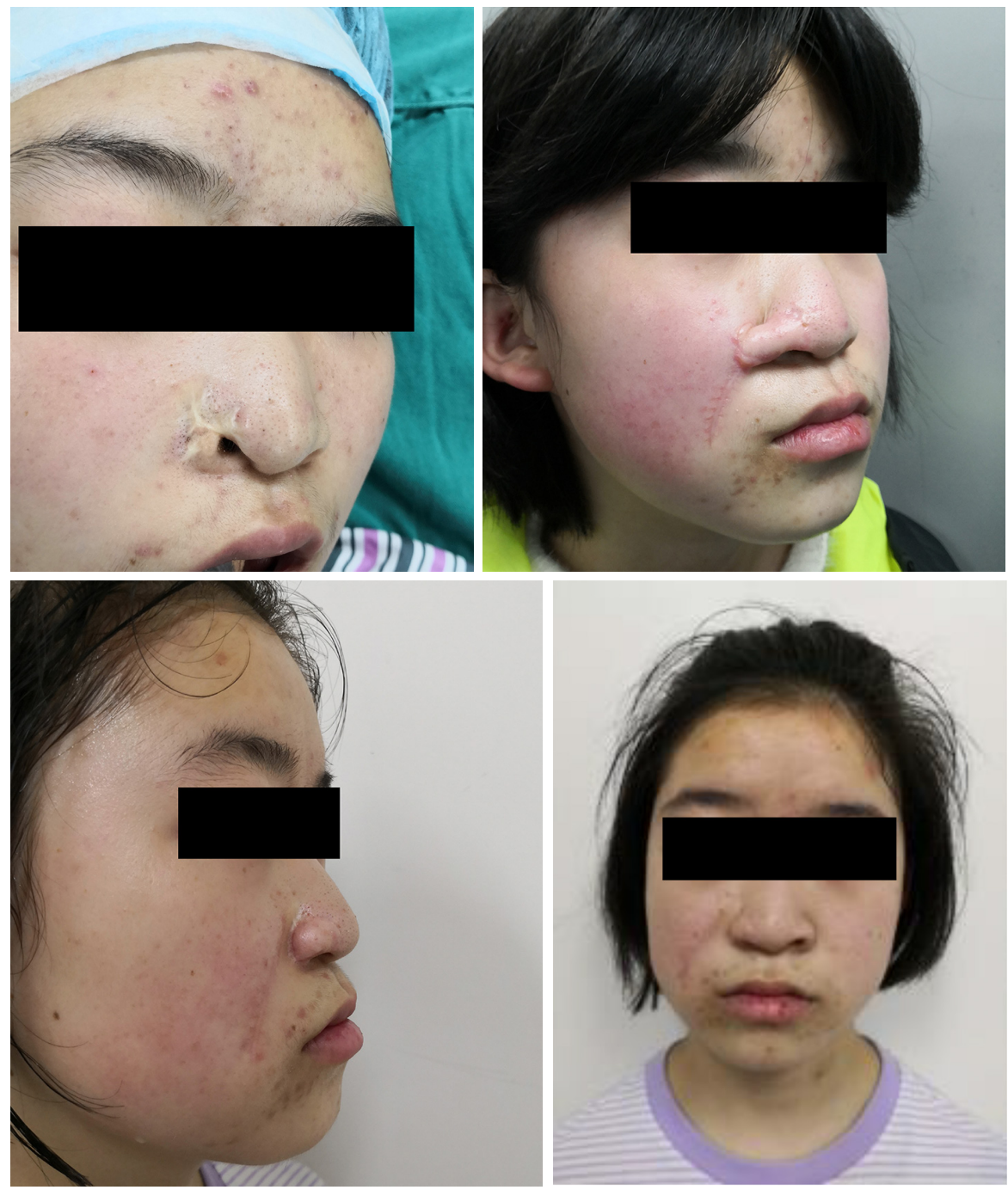

Figure 3 A 19-year-old woman with right alar defects caused by animal bite that occurred when she was 4 months old. Because of limitations in medical conditions, the wound did not undergo normal treatment, and it was completely healed by scar growth and contracture formation. Full-thickness defect with contracture scar (top left). Restoration through nasolabial pedicled flap with composite cartilage grafts and the donor ear (top right). Surgical outcomes with distinct nasofacial angle and alar groove at 8 months after pedicle amputation (bottom).

In order to achieve an ideal and long-lasting esthetic appearance, the cartilage and skin harvested from the conchal cavity were used to rebuild a stable cartilage support and smooth the alar line. For Asian patients, the supporting cartilage graft is crucial in preventing the retraction of the alar rim (16). Although the free auricular composite graft is commonly used to correct alar defects, the size of the graft should be no more than $1.0 \mathrm{~cm} \times 1.5 \mathrm{~cm}$ due to the lack of a stable vascular supply (17). Therefore, this method is not suitable to repair the severe alar defects. Considering the factors influencing auricular shape, ear asymmetry often results when the length of the harvested composite tissue $>1.5 \mathrm{~cm}$. In our cases, the application of composite conchal grafts not only avoided damage to the auricular shape but also capitalized on the curl of the conchal cartilage to provide the natural radian of the nasal ala. Furthermore, the anterior conchal skin was harvested together with cartilage to reconstruct the inner layer of nasal ala, which means less skin was needed from the skin flap, thus avoiding longer donor site scars. The range of harvested skin from the ear was smaller than that of the cartilage, and the alar rim was rebuilt with a pedicled flap to ensure a rounded contour and 


\section{Page 8 of 9}

any possible scar hidden by the nostril. The donor auricle remained unchanged in terms of shape after appropriate repair and all incisions were well concealed. Compared to simple wrapping of free cartilage with perforator flaps, the ala reconstructed with our technique appeared thinner and did not need to undergo flap-thinning procedures (18). With the help of stable and sufficient blood supply from pedicled flaps, all the conchal composite grafts completely survived, with no atrophy detected during the long-time follow-up period.

Although our patients underwent 2 or 3 stages of operations, all procedures were essential to ensure decent outcomes in severe and large traumatic alar defects. Surgical outcomes were satisfactory and acceptable, as evaluated blindly by certified plastic surgeons. All patients achieved excellent contour restoration and uniform color, without sacrificing auricular and facial symmetry.

This study has some limitations. All treatment experiences came from a single institution. The findings of the study cannot be generalized to other ethnicities, due to Asians typically having thicker and more compact dermis than Europeans. This study involved a limited number of cases; thus, additional cases are needed in future clinical studies in order to further confirm the effectiveness of our reported approach. A multicenter study should be performed to increase the credibility of our research.

In conclusion, a pedicled flap combined with conchal composite grafts should be considered for the treatment of severe traumatic nasal alar defect, as it provides a uniform skin color, thin internal layer, stable cartilage support, rounded rim, and retains the nasofacial angle. This is a reproducible technique that enables a predictably adequate outcome for severe traumatic nasal ala defects, especially in Asian patients.

\section{Acknowledgments}

Funding: This work was supported by grants from the National Natural Science Foundation of China (81871574), Sichuan Science and Technology Program (2017JY0335, 2017FZ0055 and 2017JY0250), and Scientific Research Projects of Sichuan Health Commission (19PJ097).

\section{Footnote}

Reporting Checklist: The authors have completed the STROBE reporting checklist. Available at http://dx.doi. org/10.21037/atm-20-6454
Zhang et al. Reconstruction of severe traumatic nasal alar defects

Data Sharing Statement: Available at http://dx.doi. org/10.21037/atm-20-6454

Conflicts of Interest: All authors have completed the ICMJE uniform disclosure form (available at http://dx.doi. org/10.21037/atm-20-6454). The authors have no conflicts of interest to declare.

Ethical Statement: The authors are accountable for all aspects of the work in ensuring that questions related to the accuracy or integrity of any part of the work are appropriately investigated and resolved. Informed consent was provided by all patients preoperatively. The study was approved by the Clinical Research Ethics Committee of West China Hospital of Sichuan University. All procedures performed in this study involving human participants were in accordance with the Declaration of Helsinki (as revised in 2013).

Open Access Statement: This is an Open Access article distributed in accordance with the Creative Commons Attribution-NonCommercial-NoDerivs 4.0 International License (CC BY-NC-ND 4.0), which permits the noncommercial replication and distribution of the article with the strict proviso that no changes or edits are made and the original work is properly cited (including links to both the formal publication through the relevant DOI and the license). See: https://creativecommons.org/licenses/by-nc-nd/4.0/.

\section{References}

1. Goldberg LH, Kimyai-Asadi A, Silapunt S. "Jigsaw puzzle" advancement flap for repair of a surgical defect involving the lateral nasal ala. Dermatol Surg 2005;31:569-71.

2. Constantine FC, Lee MR, Sinno S, et al. Reconstruction of the nasal soft triangle subunit. Plast Reconstr Surg 2013;131:1045-50.

3. Konofaos P, Alvarez S, McKinnie JE, et al. Nasal Reconstruction: A Simplified Approach Based on 419 Operated Cases. Aesthetic Plast Surg 2015;39:91-9.

4. Lin W, Qing Y, Liu J, et al. Alar flap combined with free auricular composite flap for the reconstruction of nasal alar defect. J Craniofac Surg 2015;26:562-4.

5. Parrett BM, Pribaz JJ. An algorithm for treatment of nasal defects. Clin Plast Surg 2009;36:407-20.

6. Qian C, Yaodong X, Xiaoming H, et al. Repair of fullthickness alar defects. Dermatol Surg 2012;38:1639-44.

7. Prakash S, Panda R, Kumar V, et al. Nasolabial Perforator 
Flap for One-stage Reconstruction of Nasal Defects. J Cutan Aesthet Surg 2017;10:22-7.

8. Sanniec K, Malafa M, Thornton JF. Simplifying the Forehead Flap for Nasal Reconstruction: A Review of 420 Consecutive Cases. Plast Reconstr Surg 2017;140:371-80.

9. Funayama E, Yamamoto Y, Furukawa H, et al. FullThickness Entire Nasal Alar Reconstruction Using a Forehead Flap in Asians: No Cartilaginous Infrastructural Lining Is Necessary. J Craniofac Surg 2017;28:734-7.

10. Yotsuyanagi T, Yamashita K, Urushidate S, et al. Nasal reconstruction based on aesthetic subunits in Orientals. Plast Reconstr Surg 2000;106:36-44; discussion 45-6.

11. Lindsay KJ, Morton JD. Flap or graft: The best of both in nasal ala reconstruction. J Plast Reconstr Aesthet Surg 2015;68:1352-7.

12. Albertini JG, Hansen JP. Trilobed flap reconstruction for distal nasal skin defects. Dermatol Surg 2010;36:1726-35.

13. Xue CY, Li L, Guo LL, et al. The bilobed flap for reconstruction of distal nasal defect in Asians. Aesthetic

Cite this article as: Zhang Z, Cheng L, Huang TCT, Hu H, Liu R, Pu Y, Wang R, Li Z, Chen J, Cen Y, Liang G, Qing Y. Repair of severe traumatic nasal alar defects with combined pedicled flap and conchal cartilage composite grafts: a retrospective study. Ann Transl Med 2020;8(22):1495. doi: 10.21037/atm-20-6454
Plast Surg 2009;33:600-4.

14. Ascari-Raccagni A, Dondas A, Righini M, et al. The eastwest advancement flap (horizontal advancement flap) to repair a defect on the nose ala. J Eur Acad Dermatol Venereol 2010;24:926-9.

15. Cvancara JL, Wentzell JM. Shark island pedicle flap for repair of combined nasal ala-perialar defects. Dermatol Surg 2006;32:726-9.

16. Han DH, Mangoba DC, Lee DY, et al. Reconstruction of nasal alar defects in asian patients. Arch Facial Plast Surg 2012;14:312-7.

17. Shenaq SM, Dinh TA, Spira M. Nasal alar reconstruction with an ear helix free flap. J Reconstr Microsurg 1989;5:63-7.

18. Durgun M, Özakpınar HR, Selçuk CT, et al. Repair of Full-Thickness Nasal Alar Defects Using Nasolabial Perforator Flaps. Ann Plast Surg 2015;75:414-7.

(English Language Editor: J. Jones) 